\title{
Commentary: Rethinking fast and slow based on a critique of reaction-time reverse inference
}

\author{
Gordon Pennycook ${ }^{1 *}$, Jonathan A. Fugelsang ${ }^{1}$, Derek J. Koehler ${ }^{1}$ and \\ Valerie A. Thompson ${ }^{2}$ \\ ${ }^{1}$ Department of Psychology, University of Waterloo, Waterloo, ON, Canada, ${ }^{2}$ Department of Psychology, University of \\ Saskatchewan, Saskatoon, SK, Canada
}

Keywords: dual process theories, response time, reaction time, reasoning, decision making

\section{A commentary on}

Rethinking fast and slow based on a critique of reaction-time reverse inference by Krajbich, I., Bartling, B., Hare, T., and Fehr, E. (2015). Nat. Commun. 6:7455. doi: 10.1038/ncomms8455

\section{OPEN ACCESS}

Edited by:

Ulrich Hoffrage,

University of Lausanne, Switzerland

Reviewed by:

Wim De Neys,

Centre National de la Recherche

Scientifique, France

Johannes Lohse,

University of Heidelberg, Germany

*Correspondence:

Gordon Pennycook

gpennyco@uwaterloo.ca

Specialty section:

This article was submitted to

Cognition,

a section of the journal

Frontiers in Psychology

Received: 12 March 2016

Accepted: 25 July 2016

Published: 05 August 2016

Citation:

Pennycook G, Fugelsang JA, Koehler DJ and Thompson VA (2016)

Commentary: Rethinking fast and

slow based on a critique of reaction-time reverse inference.

Front. Psychol. 7:1174.

doi: 10.3389/fpsyg.2016.01174
An increasingly common claim among cognitive psychologists is that the human mind is capable of two fundamentally different types of processes (Evans and Stanovich, 2013): Type 1 processing that is triggered autonomously by a stimulus and Type 2 processing that operates on a deliberate level via working memory and that allows for decoupling or override from default (Type 1) outputs. An article recently published in Nature Communications by Krajbich et al. (2015; hereafter, KBHF) focused on the use of response time (RT) differences as evidence for these dual-process theories (hereafter, DPT). KBHF outline how some dual-process theorists argue that if RTs are shorter for some response ("A") than some other response ("B"), then this supports a DPT wherein Response A is intuitive and Response $B$ is deliberative. Then, using economic games and intertemporal choice as examples, KBHF go on to argue that these RT differences can be better accounted for by sequential sampling models (SSM). Specifically, they argue that RT should increase when participants are presented options that are hard to discriminate (e.g., between two equally preferential choices). Thus, SSMs highlight the importance of conflict between choices as an explanation for RT differences. KBHF conclude by strongly cautioning against the use of RT differences to support DPTs.

In this commentary we will briefly discuss two important questions that arise from KBHF's important contribution: (1) Are all cases in which researchers have used RTs to support DPTs undermined by KBHF's analysis? and (2) Are there any RT differences that are more easily explained under DPT than by SSMs? Our overarching goal is to illustrate that the scope of KBHF's analysis is far more limited than would be reasonable surmised by their original article.

\section{ARE ALL CASES IN WHICH RESEARCHERS HAVE USED RTS TO SUPPORT DPTS UNDERMINED BY KBHF'S ANALYSIS?}

As mentioned, KBHF focus on DPTs in which responses are labeled as either "intuitive" or "deliberative." KBHF accurately demonstrate the problematic nature of inferring that a given response is intuitive or deliberative based on fast or slow RTs (respectively). Indeed, in dual-process research, this practice is known as the labeling fallacy and most commonly occurs in the domain of normative accuracy (with logical responses assumed to be the result of Type 2 processing; e.g., Gibbard, 1990; Epstein, 1994). Crucially, however, this practice has been decried by dual-process 
theorists (e.g., Evans and Stanovich, 2013), who point out that Type 1 processing can lead to correct responses and Type 2 processing can support biased processing (Evans, 2007; Stanovich, 2011). Unfortunately, KBHF do not mention that there are many DPTs that do not attempt to make such claims. For example, Evans and Stanovich (2013) have claimed that speed and accuracy are correlated but not central factors in what determines the distinction between Type 1 and Type 2 processes. This is an important addendum to KBHF because, based on their analysis alone, it is unclear whether there is good evidence to contend that RT differences should not be used to support any form of DPT. They fail to mention that there are any other classes of DPT outside of the limited class that they discuss.

\section{ARE THERE ANY RT DIFFERENCES THAT ARE MORE EASILY EXPLAINED UNDER DPT THAN BY SSMs?}

That KBHF only discussed one class of DPT does not mean that other classes of DPT are necessarily immune from their criticism. Thus, we will highlight a set of empirical results that are as easily or perhaps even more easily explained under DPTs than by simple SSMs. Our goal is to illustrate not simply that the DPT that KBHF outlined is not representative of the broader literature, but that there is good reason to believe that RTs can be a useful tool in DPT research.

Recent DPTs have focused on the conflict between evidence (or, put differently, response outputs) as a mechanism that causes deliberation and, as a result, RT differences (De Neys, 2012, 2014; Sloman, 2014; Handley and Trippas, 2015; Pennycook et al., 2015; for an important predecessor, see Sloman, 1996; for an example in the realm of cooperation, see Evans et al., 2015) ${ }^{1}$. Thus, like SSMs, there are DPTs that focus on response competition (manipulated experimentally) and not on labeling responses as intuitive or deliberative. Consider, for example, research on baserate problems. These problems describe a conflict between the probability of group membership based on a base-rate (e.g., 99.5\% chance that Paul is a nurse) and a set of stereotypes that are consistent with an alternative (e.g., Paul seems more like a doctor). The typical finding for problems of this type is that people have a strong preference for the stereotypical information (e.g., $81 \%$ stereotypical responses in De Neys and Glumicic, 2008, Experiment 1). Nonetheless, for example, De Neys and Glumicic found that participants took (roughly) 5 additional seconds to give stereotypical responses if they were presented conflicting base-rate information (relative to nonconflict versions of the problems). How can this RT difference be explained?

Based on KBHF's characterization of DPT, the conclusion from DPT proponents would be that stereotypes are "Type 1" responses whereas base-rates are "Type 2" responses. This,

${ }^{1}$ The underlying claim is that an increase in RT indicates a potential increase in deliberative Type 2 processing. Notably, this is not the same as arguing that longer RTs automatically indicate the presence of Type 2 processes (and implied absence of Type 1 processing). That is, a relative increase in Type 2 processing should be reflected in longer RTs, but this does not mean that the associated response was not initially generated autonomously and intuitively. however, is not the case. De Neys and Glumicic's (2008) primary comparison (with respect to RTs) was not between base-rate and stereotypical responses, but between incongruent (conflict) and congruent (no-conflict) problems. Specifically, they found that participants take longer when solving problems that contain a conflict between base-rate and stereotypes regardless of whether they give base-rate or stereotypical responses. This finding, first reported using RTs, was then conceptually replicated using a wide variety of different tasks (e.g., syllogisms; conditionals; the conjunction fallacy; and ratio bias) and additional measures (e.g., memory recall; verbal protocols; skin conductance response; confidence; fMRI; and ERP; see De Neys, 2012, 2014 for reviews). This supports the contention that the detection of conflict between initial response outputs causes Type 2 processing (Pennycook et al., 2015).

We should note that the increase in RT as a function of the conflict manipulation can be accommodated under SSMs. Indeed, KBHF's key demonstration involved a manipulation of choice conflict. Both SSMs and DPTs predict that RT should increase when two choices come into conflict. However, in our view, understanding this conflict at the cognitive level implicates dual-processing. For example Pennycook et al. (2015) argued for a three-stage dual-process model in which conflicting initial choice outputs (Stage 1) may be recognized as such (Stage 2 ), which then initiates analytic processing (Stage 3 ). This goes beyond the simple observation that choice conflict causes increased RT.

Importantly, not all patterns of data are equally easy to accommodate under DPTs and SSMs (but see Alos-Ferrer, 2016 for a dual-process drift diffusion model). For example, although De Neys and Glumicic (2008) found evidence for an increase in RT for stereotypical responses to conflict vs. no-conflict problems with extreme base-rates (e.g., 995 lawyers, 5 engineers), this effect diminishes if base-rates are made moderate (e.g., 700 lawyers, 300 engineers; Pennycook et al., 2012, 2015). In theory, this could be accommodated by a SSM wherein participants experience more conflict between stereotypical choices and extreme baserates than they do with moderate base-rates. Crucially, however, manipulating the extremity of the base-rates does not have an effect on RTs for base-rate responses to conflict problems (i.e., the base-rate extremity effect is only evident for stereotypical responses; Pennycook et al., 2012, 2015).

According to Pennycook et al. (2015), this pattern of results occurs because RT for base-rate responses is primarily determined by cognitive decoupling-that is, the time it takes to override the salient stereotype via Type 2 processing (the stereotypes are identical across moderate and extreme baserate experiments). The stereotypical responses are fundamentally different because at least some of the people who do not spend much time giving stereotypical answers genuinely do not distinguish between conflict and non-conflict problems (i.e., they are not detecting the conflict between their stereotypical answer and presented base-rates). Pennycook et al. (2015) argued that the probability of this happening increases when base-rates are made moderate relative to extreme, hence the aforementioned pattern of results. Thus, RTs can be used in an experimental context to distinguish between conflict detection and cognitive 
decoupling in DPTs. At the very least, evidence of RT differences as a consequence of response conflict is not necessarily evidence for SSMs and against DPTs.

\section{CONCLUSION}

In our opinion, there are good reasons to continue using RTs in DPT research. We have focused on one example, but there are other types of evidence to support this contention. For example, Thompson and colleagues (Thompson et al., 2011, 2013; Thompson and Johnson, 2014) found a negative correlation between the "feeling of rightness" associated with an initial response (e.g., stereotypical responses for base-rate problems) and the amount of time spent reasoning when giving a final answer. This finding indicates that metacognitive processes are an important determinant of how much Type 2 processing will be engaged for any given sort of problem. In a different realm altogether, there is evidence that religious believers are less analytic (see Pennycook et al., 2016) and, as a consequence, spend less time when presented with reasoning problems (Pennycook et al., 2013, 2014). These findings were predicted by DPTs, which

\section{REFERENCES}

Alos-Ferrer, C. (2016). A dual-process diffusion model. J. Behav. Decis. Mak. doi: 10.1002/bdm.1960. [Epub ahead of print].

De Neys, W. (2012). Bias and conflict: a case for logical intuitions. Perspect. Psychol. Sci. 7, 28-38. doi: 10.1177/1745691611429354

De Neys, W. (2014). Conflict detection, dual processes, and logical intuitions: some clarifications. Think. Reason. 20, 167-187. doi: 10.1080/13546783.2013.8 54725

De Neys, W., and Glumicic, T. (2008). Conflict monitoring in dual process theories of thinking. Cognition 106, 1248-1299. doi: 10.1016/j.cognition.2007. 06.002

Epstein, S. (1994). Integration of the cognitive and psychodynamic unconscious. Am. Psychol. 49, 709-724. doi: 10.1037/0003-066X.49.8.709

Evans, A. M., Dillon, K. D., and Rand, D. G. (2015). Fast but not intuitive, slow but not reflective: decision conflict drives reaction times in social dilemmas. J. Exp. Psychol. 144, 951-966. doi: 10.1037/xge00 00107

Evans, J. St. B. T. (2007). Hypothetical Thinking: Dual-Processes in Reasoning and Judgment. New York, NY: Psychology Press.

Evans, J. St. B. T., and Stanovich, K. E. (2013). Dual-process theories of higher cognition advancing the debate. Perspect. Psychol. Sci. 8, 223-241. doi: $10.1177 / 1745691612460685$

Gibbard, A. (1990). Wise Choices, Apt Feelings: A Theory of Normative Judgment. Cambridge, MA: Harvard University Press.

Handley, S. J., and Trippas, D. (2015). Dual processes, knowledge, and structure: a critical evaluation of the default interventionist account of biases in reasoning and judgment. Psychol. Learn. Motiv. 62, 2-24.

Krajbich, I., Bartling, B., Hare, T., and Fehr, E. (2015). Rethinking fast and slow based on a critique of reaction-time reverse inference. Nat. Commun. 6:7455. doi: $10.1038 /$ ncomms 8455

Pennycook, G., Cheyne, J. A., Barr, N., Koehler, D. J., and Fugelsang, J. A. (2014). Cognitive style and religiosity: the role of conflict detection. Mem. Cognit. 42, 1-10. doi: 10.3758/s13421-013-0340-7

Pennycook, G., Cheyne, J. A., Koehler, D. J., and Fugelsang, J. A. (2013). Belief bias during reasoning among religious believers and skeptics. Psychon. Bull. Rev. 20, 806-811. doi: 10.3758/s13423-013-0394-3 indicates that the framework has been useful for generating hypotheses to be tested using RTs.

Dual-process theory has proven to be quite popular among psychologists. Naturally, however, not all applications of DPT are created equal. Dual-process research that relies on experimental manipulations and that is not focused on making claims about whether responses are purely "intuitive" or "analytic" are (at least) on the same footing as other decision making models. Further, empirical work that teases apart the cognitive mechanisms that explain why decisions slow in the face of conflicting evidence is required, but in our view DPTs are in a strong position to facilitate this research.

\section{AUTHOR CONTRIBUTIONS}

GP wrote the article. VT, DK, and JF provided critical revisions.

\section{FUNDING}

The primary author was supported by a Canada Graduate Scholarship from the Natural Sciences and Engineering Research Council of Canada.

Pennycook, G., Fugelsang, J. A., and Koehler, D. J. (2012). Are we good at detecting conflict during reasoning? Cognition 124, 101-106. doi: 10.1016/j.cognition.2012.04.004

Pennycook, G., Fugelsang, J. A., and Koehler, D. J. (2015). What makes us think? A three-stage dual-process model of analytic engagement. Cogn. Psychol. 80, 34-72. doi: 10.1016/j.cogpsych.2015.05.001

Pennycook, G., Ross, R. M., Koehler, D. J., and Fugelsang, J. A. (2016). Atheists and agnostics are more reflective than religious believers: four empirical studies and a meta-analysis. PLoS ONE 11:e0153039. doi: 10.1371/journal.pone.0153039

Sloman, S. A. (1996). The empirical case for two systems of reasoning. Psychol. Bull. 119, 3-22. doi: 10.1037/0033-2909.119.1.3

Sloman, S. A. (2014). "Two systems of reasoning: an update," in Dual Process Theories of the Social Mind, eds J. Sherman, B. Gawronski, and Y. Trope (New York, NY: Guilford Press).

Stanovich, K. E. (2011). Rationality and the Reflective Mind. New York, NY: Oxford University Press.

Thompson, V. A., and Johnson, S. C. (2014). Conflict, metacognition, and analytic thinking. Think. Reason. 20, 215-244. doi: 10.1080/13546783.2013.869763

Thompson, V. A., Prowse Turner, J. A., and Pennycook, G. (2011). Intuition, metacognition, and reason. Cogn. Psychol. 63, 107-140. doi: 10.1016/j. cogpsych.2011.06.001

Thompson, V. A., Prowse Turner, J., Pennycook, G., Ball, L., Brack, H., Ophir, Y., et al. (2013). The role of answer fluency and perceptual fluency as metacognitive cues for initiating analytic thinking. Cognition 128, 237-251. doi: 10.1016/j.cognition.2012.09.012

Conflict of Interest Statement: The authors declare that the research was conducted in the absence of any commercial or financial relationships that could be construed as a potential conflict of interest.

Copyright (C) 2016 Pennycook, Fugelsang, Koehler and Thompson. This is an openaccess article distributed under the terms of the Creative Commons Attribution License (CC BY). The use, distribution or reproduction in other forums is permitted, provided the original author(s) or licensor are credited and that the original publication in this journal is cited, in accordance with accepted academic practice. No use, distribution or reproduction is permitted which does not comply with these terms. 